\title{
Parity-locking effect in a strongly-correlated ring
}

\author{
C. A. Stafford ${ }^{1}$ and D. F. Wang ${ }^{2}$ \\ ${ }^{1}$ Institut de Physique Théorique, Université de Fribourg \\ CH-1700 Fribourg, Switzerland \\ ${ }^{2}$ Institut de Physique Théorique, EPF Lausanne \\ CH-1015 Lausanne, Switzerland
}

December 5, 2017

\begin{abstract}
Orbital magnetism in an integrable model of a multichannel ring with long-ranged electron-electron interactions is investigated. In a noninteracting multichannel system, the response to an external magnetic flux is the sum of many diamagnetic and paramagnetic contributions, but we find that for sufficiently strong correlations, the contributions of all channels add constructively, leading to a parity (diamagnetic or paramagnetic) which depends only on the total number of electrons. Numerical results confirm that this parity-locking effect is robust with respect to subband mixing due to disorder.
\end{abstract}

The free energy $F(\phi)$ of a metallic ring threaded by an Aharonov-Bohm flux $(\hbar c / e) \phi$ is a periodic function of $\phi$, with period $2 \pi$ [1]]. The system is said to be diamagnetic if $F$ is minimal for $\phi=0$, and paramagnetic if $F$ is minimal for $\phi=\pi$ (1/2 flux quantum). A purely one-dimensional (1D) ring with $N$ spinless electrons is diamagnetic if $N$ is even and paramagnetic if $N$ is odd, independent of disorder and interactions [2, 3]. This connection between the parity of $N$ and the sign of the magnetic response of the system is sometimes referred to as Leggett's theorem [2]. The persistent current $I=-(e / \hbar) \partial F / \partial \phi$ is a periodic function of $\phi$ with amplitude $I_{0}=e v_{F} / L$ in a clean $1 \mathrm{D}$ ring $\left[4\right.$, where $v_{F}$ is the Fermi velocity and $L$ the circumference of the ring. In a ring with many independent channels, $I$ is the sum of many such diamagnetic and paramagnetic contributions, and thus has a random sign and small amplitude [1]. Leggett's theorem is thus generally violated in multichannel systems.

In an interacting system, the parities of different channels are no longer independent. In this article, we investigate the orbital magnetism of 1D $S U(M)$ fermions interacting via the potential $V(x)=g / x^{2}$. We find that for $g>0$, Leggett's theorem is restored for an arbitrary number of channels $M$. In addition, provided the ring is sufficiently thin $\left(k_{F} L>2 \pi M\right)$ we find that the 
magnetic response of all channels add constructively, leading to a large enhancement of the persistent current. A disordered two-channel ring with interchannel interactions is also investigated numerically, and shows, importantly, that the parity-locking effect persists even when the subbands are mixed strongly by disorder. Interchannel correlations of this sort may be important to explain the anomalously large observed value of the persistent current in normal metal rings [5. 6.

Interacting spinless electrons in a non-disordered ring with $M$ transverse channels, threaded by an Aharanov-Bohm flux $(\hbar c / e) \phi$, may be represented by 1D $S U(M)$ fermions. The transverse degrees of freedom may be represented by an $S U(M)$ spin variable $\sigma=1, \ldots, M$. In the absence of disorder, and for interactions which depend only on the electrons' coordinates along the ring (i.e., for thin rings), the number of electrons $K_{\sigma}$ in each channel is conserved. The Hamiltonian of the system is

$$
H=-\frac{1}{2} \sum_{i=1}^{N} \frac{\partial^{2}}{\partial x_{i}^{2}}+\sum_{i<j} V\left(x_{i}-x_{j}\right)+\sum_{\sigma=1}^{M} K_{\sigma} \varepsilon_{\sigma},
$$

where $N=\sum_{\sigma} K_{\sigma}$ is the total number of electrons and $\varepsilon_{\sigma}$ is the energy minimum of subband $\sigma$. Units with $\hbar=m=1$ are used. The Aharanov-Bohm flux leads to the twisted boundary condition [1]

$$
\Psi\left(x_{1} \sigma_{1}, \cdots,\left(x_{i}+L\right) \sigma_{i}, \cdots, x_{N} \sigma_{N}\right)=e^{i \phi} \Psi\left(x_{1} \sigma_{1}, \cdots, x_{i} \sigma_{i}, \cdots, x_{N} \sigma_{N}\right) .
$$

For simplicity, let us consider equally spaced subbands $\varepsilon_{\sigma+1}-\varepsilon_{\sigma}=\Delta \equiv E_{F} / M$. The subband splitting $\Delta$ plays the role of an $S U(M)$ magnetic field. As we shall see, the effect of repulsive interactions is to renormalize this effective field, causing a condensation of electrons into the lowest subband. At $T=0$, the equilibrium persistent current is given by $I(\phi)=-(e / \hbar) \partial E_{0} / \partial \phi$, where $E_{0}(\phi)$ is the ground state energy of Eq. (11), subject to the boundary condition (2).

The first harmonic of the persistent current may be characterized by the value of $I$ at $\phi=\pi / 2$ (1/4 flux quantum), assuming the higher odd harmonics are small. For $V(x)=0$, one finds

$$
I\left(\phi_{0} / 4\right)=\frac{e \hbar \pi}{2 m L^{2}} \sum_{\sigma=1}^{M}(-1)^{K_{\sigma}} K_{\sigma} .
$$

For $M \gg 1$, this leads to the well-known [7] result $|I| \sim M^{1 / 2} I_{0}$ due to the random parities of the different channels. The system may be either diamagnetic or paramagnetic, depending on the channel occupancies $K_{\sigma}$.

Let us next consider a model with long-range interactions: $V(x)=g / d(x)^{2}$, where $d(x)=(L / \pi)|\sin (\pi x / L)|$ is the chord length along the ring. This model was introduced and solved by Sutherland [8] for the case $M=1$ and $\phi=0$. First, we show that the $S U(M)$ model with twisted boundary conditions is 
completely integrable. Let us introduce the following generalized momentum operators

$$
\pi_{j}=p_{j}-i \lambda \frac{\pi}{L} \sum_{k(\neq j)} \cot \frac{\left(x_{j}-x_{k}\right) \pi}{L} P_{j k}
$$

where the operator $P_{j k}$ permutes the isospins and the positions of the two particles $j$ and $k$ simultaneously, and $\lambda=\sqrt{g+1 / 4}+1 / 2$. These generalized momentum operators are hermitian, $\pi_{i}=\pi_{i}^{\dagger}$. The Hamiltonian can be expressed as a quadratic form in terms of the $\pi_{i}$,

$$
H=\sum_{i=1}^{N} \frac{1}{2} \pi_{i}^{2}+\sum_{\sigma=1}^{M} \epsilon_{\sigma} K_{\sigma}+\text { const. }
$$

Introducing a set of new operators $\tilde{\pi}_{i}=\pi_{i}+\lambda \sum_{j(\neq i)} P_{i j}$, one can show that the following infinite number of physical operators commute with each other

$$
\left[\tilde{I}_{n}, \tilde{I}_{m}\right]=0,
$$

where $\tilde{I}_{n}=\sum_{i=1}^{N}\left(\tilde{\pi}_{i}\right)^{n}$, with $n=0,1,2, \ldots, \infty$. Furthermore, one can show that these operators also commute with the Hamiltonian

$$
\left[H, \tilde{I}_{n}\right]=0 .
$$

This therefore provides a proof of the complete integrability of the system. Our proof of integrability follows that given by Polychronakos for the CalogeroSutherland model [9]. Note, however, that the operator $P_{i j}$ permutes the positions and the isospins of two particles $i$ and $j$, while the operator $M_{i j}$ of Ref. [9] only exchanges the positions of the two particles.

Consider the following Jastrow wavefunction for given channel occupancies $K_{\sigma}$

$$
\tilde{\Psi}_{0}\left(x_{1} \sigma_{1}, x_{2} \sigma_{2}, \cdots, x_{N} \sigma_{N}\right)=\prod_{1 \leq i<j \leq N}\left|\sin \left(\frac{x_{i}-x_{j}}{L} \pi\right)\right|^{\lambda-1} \sin \left(\frac{x_{i}-x_{j}}{L} \pi\right),
$$

where the isospins of the particles are symmetric under exchange. This wavefunction satisfies periodic boundary conditions $(\phi=0)$ if $N$ is odd and satisfies antiperiodic boundary conditions $(\phi=\pi)$ if $N$ is even. It also satisfies FermiDirac statistics. Furthermore, this wavefunction is annihilated by the operators $\pi_{i}$, with $i=1,2, \cdots, N$ :

$$
\pi_{i} \tilde{\Psi}_{0}\left(x_{1} \sigma_{1}, x_{2} \sigma_{2}, \cdots, x_{N} \sigma_{N}\right)=0
$$

which indicates that it is the ground state of $H$ in the subspace with fixed $K_{\sigma}$. The absolute ground state of the system thus occurs for $\phi=0$ when $N$ is odd and for $\phi=\pi$ when $N$ is even, regardless of the subband spacing $\varepsilon_{\sigma}$. The 
Leggett theorem thus holds for this interacting multichannel system, i.e., the ground state is diamagnetic if $N$ is odd and paramagnetic if $N$ is even.

Now, let us consider general values of the external magnetic flux. For $g>0$, the ground state is highly degenerate in the limit $L \rightarrow \infty$ in the absence of $S U(M)$ symmetry breaking $(\Delta=0)$ due to the strong repulsion of the potential at the origin, which prohibits particle exchange. In a finite ring, one expects many $S U(M)$ level crossings 10 as a function of $\phi$. These states differ in energy by at most $\pi \hbar v_{F} / L$ due to boundary effects; all electrons will thus be condensed into the lowest subband for $\Delta>\pi \hbar v_{F} / L$, i.e., for $k_{F} L>2 \pi M$, which is satisfied provided the ring is sufficiently thin. The ground state of the system in this "ferromagnetic" state has the Jastrow product form

$$
\Psi(\{x\})=\exp \left(i \frac{\phi-a}{L} \sum_{k=1}^{N} x_{k}\right) \prod_{1 \leq i<j \leq N}\left|\sin \left(\frac{x_{i}-x_{j}}{L} \pi\right)\right|^{\lambda-1} \sin \left(\frac{x_{i}-x_{j}}{L} \pi\right)
$$

for $0 \leq \phi \leq \pi$, where $a=0$ if $N$ is odd and $a=\pi$ if $N$ is even. One readily verifies that $\Psi$ is an eigenstate of Eq. (11), has the correct symmetry, and obeys the twisted boundary condition (2). This state coincides with the absolute ground state $\tilde{\Psi}_{0}$ for $\phi=0(\pi)$ when $N$ is odd (even). The ground state energy is found to be

$$
E_{0}(\phi)=\frac{\pi^{2}(\lambda+1)^{2} N\left(N^{2}-1\right)}{6 L^{2}}+\frac{N}{2}\left(\frac{\phi-a}{L}\right)^{2} .
$$

The corresponding persistent current is

$$
I\left(\phi_{0} / 4\right)=(-1)^{N} \frac{e \hbar \pi N}{2 m L^{2}} \sim(-1)^{N} M I_{0} .
$$

The condensation of all electrons into the lowest subband caused by the strong repulsive interactions thus leads to an enhancement of the typical persistent current in the ballistic regime by a factor of $M^{1 / 2}$ compared to that for noninteracting electrons, given in Eq. (3).

A peculiarity of the integrable model considered above is that the number of electrons in each channel is a constant of the motion. Both disorder and more realistic interactions which depend on the transverse coordinate will break this symmetry, and it is therefore important to verify that the parity-locking effect is not destroyed. To this end, we have considered a disordered two-channel ring, modeled in the tight-binding approximation, with a nearest-neighbor interchain interaction $V$ included to induce interchannel correlations. The Hamiltonian is

$H=\sum_{i=1}^{L}\left[\sum_{\alpha=1}^{2}\left(e^{i \phi / L} c_{i \alpha}^{\dagger} c_{i+1 \alpha}+\right.\right.$ H.c. $\left.+\varepsilon_{i \alpha} \rho_{i \alpha}\right)+\frac{\Delta}{2}\left(c_{i 1}^{\dagger} c_{i 2}+\right.$ H.c. $\left.)+V \rho_{i 1} \rho_{i 2}\right]$, 
where $c_{i \alpha}^{\dagger}$ creates a spinless electron at site $i$ of chain $\alpha, \rho_{i \alpha} \equiv c_{i \alpha}^{\dagger} c_{i \alpha}$, and $\varepsilon_{i \alpha}$ is a random number in the interval $[-\varepsilon / 2, \varepsilon / 2]$. The interchain hopping determines the subband splitting $\Delta$. Fig. 1 shows the persistent current for rings with 5 spinless electrons on 18 sites as a function of $V$, calculated using the Lanczos technique. Both the ensemble average of $I$ for 500 systems (squares) and the persistent current of a typical system (solid curve) are indicated. The error bars indicate the standard deviation $\delta I=\left(\left\langle I^{2}\right\rangle-\langle I\rangle^{2}\right)^{1 / 2}$ over the ensemble (not the statistical uncertainty in the mean value). The subband splitting $\Delta=0.8$ is chosen so that in the absence of disorder and interactions, $K_{1}=3$ and $K_{2}=2$, leading to a large cancellation of the persistent current due to the different parities of the two channels. The on-site disorder $\varepsilon=2>\Delta, E_{F}$ mixes the two channels, but does not lead to strong backscattering. For $V=0$, the sign of $I$ is essentially random, and $\langle I\rangle$ is slightly diamagnetic. As $V$ is increased, $\langle I\rangle$ oscillates in sign and increases in magnitude, becoming strongly diamagnetic for large $V .|\langle I\rangle|$ is increased by a factor of 10 as $V$ is increased from 0 to 20, while $\sqrt{\left\langle I^{2}\right\rangle}$ is increased by a factor of 3 . The system exhibits parity locking for large $V$.

While the subband occupancies are no longer constants of the motion in Eq. (13), there is a corresponding topological invariant in the disordered system, namely, the number of transverse nodes in the many-body wavefunction [2] (i.e., nodes which encircle the $\mathrm{AB}$ flux $\phi$ ). The lowest subband has no such nodes, while each electron in the second subband contributes one transverse node. In order for two electrons to pass each other as they circle the ring, such a transverse node must be present. As $V$ increases, it becomes energetically unfavorable for electrons to approach each other, so transverse nodes in the many-body wavefunction will tend to be suppressed. In the strongly-correlated limit, all such nodes will be eliminated, leading to a state whose parity depends only on the total number of electrons. In such a state, the persistent currents of all channels add constructively, leading to a large persistent current (see Fig. 1). It should be emphasized that while the enhancement of the persistent current shown in Fig. 1 is relatively modest, a much larger enhancement would be expected in a system with $M \gg 1$, based on the above arguments.

In conclusion, it has been shown that for sufficiently strong electronic correlations, the sign of the orbital magnetic response in a multichannel ring is determined solely by the parity of the total number of electrons in the system. The magnetic response of all channels add constructively, leading to a large enhancement of the persistent current. This result, which we refer to as the parity-locking effect, was demonstrated in an integrable model with long-range interactions as well as in a disordered two-channel system. It should be emphasized that the parity-locking effect holds in both ballistic and disordered systems; it is therefore complementary to mechanisms previously proposed for the enhancement of the persistent current [11, 12, 13, 14, 15], which rely on the competition between disorder and interactions. It is likely that both such mechanisms are important to explain the anomalously large observed value [5, 6] of 
the persistent current in disordered metallic rings.

C. A. S. thanks M. Büttiker for valuable discussions. This work was supported by the Swiss National Science Foundation.

\section{References}

[1] N. Byers and C. N. Yang, Phys. Rev. Lett. 7, 46 (1961).

[2] A. J. Leggett, in Granular Nanoelectronics, edited by D. K. Ferry et al. (Plenum Press, New York, 1991), p. 297.

[3] D. Loss, Phys. Rev. Lett. 69, 343 (1992).

[4] M. Buttiker, Y. Imry and R. Landauer, Phys. Lett. 96A, 365 (1983).

[5] L. P. Lévy, G. Dolan, J. Dunsmuir and H. Bouchiat, Phys. Rev. Lett. 64, 2074 (1990).

[6] V. Chandrasekhar et al., Phys. Rev. Lett. 67, 3578 (1991).

[7] H. F. Cheung, E. K. Riedel, and Y. Gefen, Phys. Rev. Lett. 62, 587 (1989).

[8] B. Sutherland, Phys. Rev. A 4, 2019 (1971); 5, 1372 (1972).

[9] A. P. Polychronakos, Phys. Rev. Lett. 69, 703 (1992).

[10] F. V. Kusmartsev, J. Phys. Condens. Matter 3, 3199 (1991); N. Yu and M. Fowler, Phys. Rev. B 45, 11795 (1992).

[11] V. Ambegaokar and U. Eckern, Phys. Rev. Lett. 65, 381 (1990).

[12] A. Schmid, Phys. Rev. Lett. 66, 80 (1991).

[13] D. Yoshioka and H. Kato, Physica B 212, 251 (1995); G. Bouzerar and D. Poilblanc, J. Physique (in press).

[14] T. Giamarchi and B. S. Shastry, Phys. Rev. B 51, 10915 (1995); H. Mori and M. Hamada, ibid. 53, 4850 (1996).

[15] R. Berkovits and Y. Avishai, Europhys. Lett. 29, 475 (1995); G. Bouzerar and D. Poilblanc, Phys. Rev. B 52, 10772 (1995); G. Chiappe, J. A. Vergés, and E. Louis, Solid State Comm. 99, 717 (1996). 


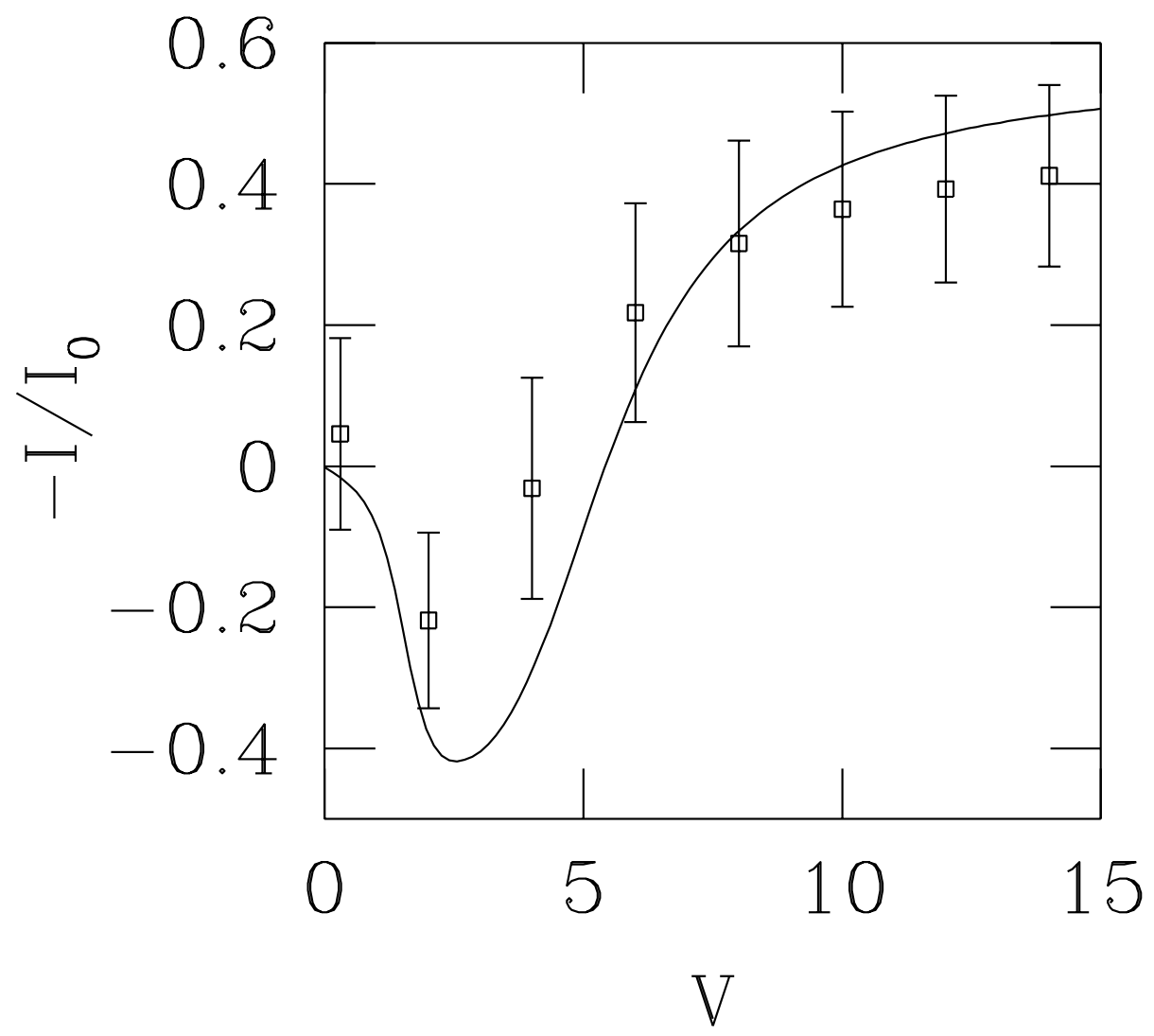

Figure 1: Persistent current $I=-(e / \hbar) \partial E_{0} /\left.\partial \phi\right|_{\phi=\phi_{0} / 4}$ of a disordered twochannel ring with 5 spinless electrons on 18 sites as a function of the interchain interaction $V$. The current is given in units of $I_{0}=e v_{F} / L$. The amplitude of the on-site disorder is $\varepsilon=2$ and the subband splitting is $\Delta=0.8$. Solid curve: persistent current for one realization of disorder. Squares: ensemble average $\langle I\rangle$ for 500 systems. The error bars indicate the width $\delta I=\left(\left\langle I^{2}\right\rangle-\langle I\rangle^{2}\right)^{1 / 2}$ of the current distribution. Note that the persistent current is diamagnetic for large $V$, as expected for a system with $N$ odd due to parity-locking; this is true for all realizations of disorder. 